\title{
Quantum mechanics with respect to different reference frames
}

\author{
L.MANGIAROTTI \\ Department of Mathematics and Informatics, University of Camerino, 62032 Camerino \\ $(M C)$, Italy \\ G.SARDANAShVILY \\ Department of Theoretical Physics, Moscow State University, 117234 Moscow, Russia
}

Geometric (Schrödinger) quantization of nonrelativistic mechanics with respect to different reference frames is considered. In classical nonrelativistic mechanics, a reference frame is represented by a connection on a configuration space fibered over a time axis $\mathbb{R}$. Under quantization, it yields a connection on the quantum algebra of Schrödinger operators. The operators of energy with respect to different reference frames are examined.

\section{INTRODUCTION}

Since reference frame transformations are time-dependent, we are in the case of timedependent nonrelativistic mechanics. A problem is that a Hamiltonian $\mathcal{H}$ of time-dependent mechanics fails to be a scalar function. Therefore, its quantization depends on a reference frame. Quantized with respect to a given reference frame $\Gamma$, a Hamiltonian $\widehat{\mathcal{H}}$ coincides with the operator $\widehat{\mathcal{E}}_{\Gamma}$ of energy relative to this reference frame, but not to other ones. Our goal is the relation (18) between operators of energy with respect to different reference frames.

A configuration space of time-dependent mechanics is a smooth real fiber bundle $\pi$ : $Q \rightarrow \mathbb{R}$, where $Q$ is an oriented manifold and $\mathbb{R}$ is a time axis. This configuration space is endowed with bundle coordinates $\left(t, q^{i}\right)$ where $t$ is a Cartesian coordinate on $\mathbb{R}$ possessing the transition functions $t^{\prime}=t+$ const. Due to these transition functions, the base $\mathbb{R}$ is provided with the standard vector field $\partial_{t}$ and the standard one-form $d t$. The velocity phase space of time-dependent mechanics is the jet manifold $J^{1} Q$ of $Q \rightarrow \mathbb{R}$ coordinated by $\left(t, q^{i}, q_{t}^{i}\right)$, and its momentum phase space is the vertical cotangent bundle $V^{*} Q$ of $Q \rightarrow \mathbb{R}$ equipped with the holonomic coordinates $\left(t, q^{i}, p_{i}=\dot{q}_{i}\right){ }^{1-3}$

A fiber bundle over $Q \rightarrow \mathbb{R}$ is always trivial. Any trivialization $Q \cong \mathbb{R} \times M$ yields a global reference frame on $Q$ as follows. A reference frame in nonrelativistic mechanics is defined as a connection

$$
\Gamma=d t \otimes\left(\partial_{t}+\Gamma^{i}\left(t, q^{j}\right) \partial_{i}\right)
$$

on the configuration bundle $Q \rightarrow \mathbb{R}^{1-5}$ Let

$$
\partial_{\Gamma}=\partial_{t}+\Gamma^{i}\left(t, q^{j}\right) \partial_{i}
$$


be the horizontal lift onto $Q$ of the standard vector field $\partial_{t}$ on $\mathbb{R}$. One can think of its values as being velocities of "observes" at points of $Q$. Accordingly, the corresponding covariant differential

$$
D_{\Gamma}: J^{1} Q \underset{Q}{\rightarrow} V Q, \quad \dot{q}^{i} \circ D^{\Gamma}=q_{t}^{i}-\Gamma^{i},
$$

determines the relative velocities with respect to the reference frame $\Gamma$. These velocities are elements of the vertical tangent bundle $V Q$ of $Q \rightarrow \mathbb{R}$ coordinated by $\left(t, q^{i}, \dot{q}_{i}\right)$. A connection $\Gamma(1)$ is called complete if the vector field $\partial_{\Gamma}(2)$ is complete. Since connections $\Gamma$ on $Q \rightarrow \mathbb{R}$ are always flat, there is one-to-one correspondence between them and the equivalence classes of atlases of $Q \rightarrow \mathbb{R}$ with time-independent transition functions. ${ }^{1,5,6}$ Namely, $\Gamma^{i}=0$ relative to bundle coordinates of an associated atlas. These coordinates are called $\Gamma$-adapted. $\Gamma$-adapted coordinates $\left(t, \bar{q}^{j}\right)$, a connection $\Gamma$ relative to arbitrary bundle coordinates $\left(t, q^{i}\right)$ reads

$$
\Gamma^{i}\left(t, q^{j}\right)=\partial_{t} q^{i}\left(t, \bar{q}^{j}\right)
$$

In particular, every trivialization of a fiber bundle $Q \rightarrow \mathbb{R}$ yields a complete connection, i.e., a reference frame on $Q$, and vice versa. We call it a global reference frame and, without a loss of generality, restrict our consideration to global reference frames.

Let $T^{*} Q$ be the cotangent bundle of a configuration space $Q$ equipped with the holonomic coordinates $\left(t, q^{i}, p, p_{i}\right)$, possessing the transition functions

$$
p_{i}^{\prime}=\frac{\partial q^{j}}{\partial q^{\prime i}} p_{j}, \quad p^{\prime}=p+\frac{\partial q^{j}}{\partial t} p_{j} .
$$

This bundle plays the role of the homogeneous momentum phase space of time-dependent mechanics. ${ }^{3,7}$ It is equipped with the canonical Liouville and symplectic forms

$$
\Xi=p d t+p_{i} d q^{i}, \quad \Omega=d \Xi=d p \wedge d t+d p_{i} \wedge d q^{i} .
$$

The corresponding Poisson bracket reads

$$
\{f, g\}_{T}=\partial^{p} f \partial_{t} g-\partial^{p} g \partial_{t} f+\partial^{i} f \partial_{i} g-\partial^{i} g \partial_{i} f, \quad f, g \in C^{\infty}\left(T^{*} Q\right) .
$$

There is the trivial one-dimensional affine bundle

$$
\zeta: T^{*} Q \rightarrow V^{*} Q
$$

It provides $V^{*} Q$ with the degenerate regular Poisson structure

$$
\{f, g\}_{V}=\partial^{i} f \partial_{i} g-\partial^{i} g \partial_{i} f, \quad f, g \in C^{\infty}\left(V^{*} Q\right),
$$

such that $\zeta$ is a Poisson morphism, i.e.,

$$
\zeta^{*}\{f, g\}_{V}=\left\{\zeta^{*} f, \zeta^{*} g\right\}_{T}
$$


Due to this fact, we can quantize time-dependent mechanics in the framework of geometric quantization of the Poisson manifold $\left(V^{*} Q,\{,\}_{V}\right) \cdot{ }^{7-9}$

A Hamiltonian on the momentum phase space $V^{*} Q$ of time-dependent mechanics is defined as a global section

$$
\mathcal{H}: V^{*} Q \rightarrow T^{*} Q, \quad p=-\mathcal{H}\left(t, q^{j}, p_{j}\right),
$$

of the affine bundle $\zeta(8)$. For instance, any connection $\Gamma(1)$ defines a Hamiltonian

$$
\mathcal{H}_{\Gamma}=\xi \circ \Gamma: Q \rightarrow J^{1} Q \rightarrow T^{*} Q, \quad \mathcal{H}_{\Gamma}=\Gamma^{i} p_{i}
$$

due to the canonical imbedding $\xi: J^{1} Q \rightarrow T^{*} Q$. A glance at the transformation law (5) shows that a Hamiltonian $\mathcal{H}(11)$ fails to be a function on $V^{*} Q$. Therefore, it can not be quantized as an element of the Poisson algebra $C^{\infty}\left(V^{*} Q\right)$ of smooth real functions on $V^{*} Q$. One can overcome this difficulty if a trivialization $Q \cong \mathbb{R} \times M$ (i.e., a reference frame) holds fixed, ${ }^{10,11}$ but must compare the results of independent quantizations with respect to distinct reference frames.

In a different way, we have performed frame independent geometric quantization of the Poisson algebra $C^{\infty}\left(T^{*} Q\right)$ on the cotangent bundle $T^{*} X{ }^{7-9}$ It contains both the subalgebra $\zeta^{*} C^{\infty}\left(V^{*} Q\right)$ and the function

$$
\left.\mathcal{H}^{*}=\partial_{t}\right\rfloor\left(\Xi-\zeta^{*} h^{*} \Xi\right)=p+\mathcal{H}
$$

which is a Hamiltonian, called the covariant Hamiltonian, on the homogeneous momentum phase space $T^{*} Q .{ }^{7,9}$ The monomorphism $\zeta^{*} C^{\infty}\left(V^{*} Q\right) \rightarrow C^{\infty}\left(T^{*} Q\right)$ is prolonged to a monomorphism of quantum algebras $\mathcal{A}_{V}$ of $V^{*} Q$ and $\mathcal{A}_{T}$ of $T^{*} Q$. These algebras consist of affine functions of momenta on $V^{*} Q$ and $T * Q$, respectively. They are represented by first order differential operators $(33)$ and $(34)$ on the $C^{\infty}(\mathbb{R})$-module $E_{R}$ of complex halfforms on $Q$ whose restriction to each fiber of $Q \rightarrow \mathbb{R}$ is of compact support. Accordingly, the covariant Hamiltonian $\mathcal{H}^{*}$, polynomial in momenta, is quantized as an element of the universal enveloping algebra of the Lie algebra $\mathcal{A}_{T}$.

A problem is that the decomposition $\mathcal{H}^{*}=p+\mathcal{H}$ and the corresponding splitting of quantum operators

$$
\widehat{\mathcal{H}^{*}}=\widehat{p}+\widehat{\mathcal{H}}=-i \partial_{t}+\widehat{\mathcal{H}}
$$

are ill defined. At the same time, any reference frame $\Gamma$ yields the decomposition

$$
\mathcal{H}^{*}=\left(p+\mathcal{H}_{\Gamma}\right)+\left(\mathcal{H}-\mathcal{H}_{\Gamma}\right)=\mathcal{H}_{\Gamma}^{*}+\mathcal{E}_{\Gamma}
$$

where $\mathcal{H}_{\Gamma}$ is the Hamiltonian (12), $\mathcal{H}_{\Gamma}^{*}$ is the corresponding covariant Hamiltonian (39), and

$$
\mathcal{E}_{\Gamma}=\mathcal{H}-\mathcal{H}_{\Gamma}=\mathcal{H}-\Gamma^{i} p_{i}
$$


is the energy relative to the reference frame $\Gamma \cdot{ }^{1-3,5,12}$ Accordingly, we obtain the splitting

$$
\widehat{\mathcal{H}}^{*}=\widehat{\mathcal{H}}_{\Gamma}^{*}+\widehat{\mathcal{E}}_{\Gamma},
$$

where $\widehat{\mathcal{E}}_{\Gamma}$ is the operator of energy relative to the reference frame $\Gamma$ and $\widehat{\mathcal{H}}_{\Gamma}^{*}(40)$ is a connection (44) on the quantum algebra $\mathcal{A}_{V}$ of $V^{*} Q$. The splitting (17) results in a desired relation

$$
\widehat{\mathcal{H}}_{\Gamma}^{*}+\widehat{\mathcal{E}}_{\Gamma}=\widehat{\mathcal{H}}_{\Gamma^{\prime}}^{*}+\widehat{\mathcal{E}}_{\Gamma^{\prime}}
$$

between operators of energy with respect to different reference frames.

Some examples are considered in Section V.

\section{CLASSICAL EVOLUTION OPERATOR AND ENERGY FUNCTIONS}

In contrast with autonomous mechanics, the Poisson structure (9) fails to provide any dynamic equation on the momentum phase space $V^{*} Q$ because Hamiltonian vector fields

$$
\vartheta_{f}=\partial^{i} f \partial_{i}-\partial_{i} f \partial^{i}, \quad f \in C^{\infty}\left(V^{*} Q\right),
$$

of functions on $V^{*} Q$ are vertical. Moreover, a Hamiltonian $\mathcal{H}$ of time-dependent mechanics is not a function on $V^{*} Q$ and, therefore, its Hamiltonian vector field with respect to the Poisson structure (9) is not defined. Dynamics of time-dependent mechanics is described as follows. ${ }^{1-3}$

A Hamiltonian $\mathcal{H}(11)$ definess the pull-back Hamiltonian form

$$
H=\mathcal{H}^{*} \Xi=p_{k} d q^{k}-\mathcal{H} d t
$$

on $V^{*} Q$, which is the well-known invariant of Poincaré Cartan. Given a Hamiltonian form $H(20)$, there exists a unique horizontal vector field $\gamma_{H}$ (i.e., $\left.\gamma_{H}\right\rfloor d t=1$ ) on $V^{*} Q$ such that $\left.\gamma_{H}\right\rfloor d H=0$. It reads

$$
\gamma_{H}=\partial_{t}+\partial^{k} \mathcal{H} \partial_{k}-\partial_{k} \mathcal{H} \partial^{k} .
$$

This vector field, called the Hamilton vector field, yields the first order Hamilton equations

$$
q_{t}^{k}-\partial^{k} \mathcal{H}=0, \quad p_{t k}+\partial_{k} \mathcal{H}=0
$$

on $V^{*} Q \rightarrow \mathbb{R}$, where $\left(t, q^{k}, p_{k}, q_{t}^{k}, p_{t k}\right)$ are coordinates on the first order jet manifold $J^{1} V^{*} Q$ of $V^{*} Q \rightarrow \mathbb{R}$. Accordingly, the Lie derivative

$$
\left.\mathbf{L}_{\gamma_{H}} f=\gamma_{H}\right\rfloor d f=\left(\partial_{t}+\partial^{k} \mathcal{H} \partial_{k}-\partial_{k} \mathcal{H} \partial^{k}\right) f
$$

of functions $f \in C^{\infty}\left(V^{*} Q\right)$ along the Hamilton vector field $\gamma_{H}(21)$ is called the evolution operator. It takes the form

$$
\mathbf{L}_{\gamma_{H}} f \approx d_{t} f=\left(\partial_{t}+q_{t}^{i} \partial_{i}+p_{t i} \partial^{i}\right) f
$$


on-shell (i.e., on the submanifold of $J^{1} V^{*} Q$ defined by the Hamilton equations (22)). If $\mathbf{L}_{\gamma_{H}}=0$, then $d_{t} f \approx 0$, i.e., a function $f$ is an integral of motion. The evolution operator takes the local form

$$
\mathbf{L}_{\gamma_{H}} f=\partial_{t} f+\{\mathcal{H}, f\}_{V},
$$

but the Poisson bracket $\{\mathcal{H}, f\}_{V}$ is ill defined because it is not a function under coordinate transformations.

In order to overcome this difficulty and quantize the evolution operator, we use the fact that a time-dependent Hamiltonian system on the momentum phase space $V^{*} Q$ can be extended to an autonomous Hamiltonian system on the homogeneous momentum phase space $T^{*} Q$ with the covariant Hamiltonian $\mathcal{H}^{*}(13){ }^{3,13,14}$ The Hamiltonian vector field of $\mathcal{H}^{*}$ with respect to the Poisson bracket $(7)$ on $T^{*} Q$ reads

$$
\gamma_{T}=\partial_{t}-\partial_{t} \mathcal{H} \partial^{p}+\partial^{k} \mathcal{H} \partial_{k}-\partial_{k} \mathcal{H} \partial^{k}
$$

It is projected onto the Hamilton vector field $\gamma_{H}(21)$ on $V^{*} Q$ so that the relation

$$
\zeta^{*}\left(\mathbf{L}_{\gamma_{H}} f\right)=\left\{\mathcal{H}^{*}, \zeta^{*} f\right\}_{T}, \quad f \in C^{\infty}\left(V^{*} Q\right),
$$

holds. In particular, a function $f \in C^{\infty}\left(V^{*} Q\right)$ is an integral of motion iff the bracket (25) vanishes.

Due to the relation (25), we can quantize the pull-back of the evolution operator (23) onto $T^{*} Q$ in the framework of geometric quantization of the symplectic manifold $\left(T^{*} Q, \Omega\right)$.

It is readily observed that the pull-back of a Hamiltonian form $H$ (20) onto the jet manifold $J^{1} V^{*} Q$ is the Poincaré-Cartan form of the Lagrangian

$$
L_{H}=h_{0}(H)=\left(p_{i} q_{t}^{i}-\mathcal{H}\right) d t, \quad h_{0}\left(d q^{i}\right)=q_{t}^{i} d t
$$

on $J^{1} V^{*} Q$, and that the Hamilton equations (22) are exactly the Lagrange equations of this Lagrangian. ${ }^{1}$ Using this fact, one can obtain conservation laws in time-dependent Hamiltonian mechanics as follows. ${ }^{1,2,5}$

Any vector field $u=u^{t} \partial_{t}+u^{i} \partial_{i}$ on a configuration space $Q$ gives rise to the vector field

$$
\widetilde{u}=u^{t} \partial_{t}+u^{i} \partial_{i}-\partial_{i} u^{j} p_{j} \partial^{i}
$$

on the momentum phase space $V^{*} Q$. The equality

$$
\mathbf{L}_{\widetilde{u}} H=\mathbf{L}_{J^{1} \widetilde{u}} L_{H}
$$

holds. Then the first variational formula applied to the Lagrangian $L_{H}$ (26) leads to the weak identity

$$
\left.\mathbf{L}_{\widetilde{u}} H \approx d_{t}(u\rfloor H\right) d t
$$


on-shell. If the Lie derivative (28) vanishes, we come to the weak conservation law $0 \approx$ $\left.d_{t}(u\rfloor H\right) d t$ of the function

$$
\left.\mathfrak{T}_{u}=u\right\rfloor d H=p_{i} u^{i}-u^{t} \mathcal{H} .
$$

By analogy with field theory, this function is called the symmetry current along $u$. Any conserved symmetry current $\mathfrak{T}_{u}(29)$ is an integral of motion.

For instance, put $u=-\partial_{\Gamma}(2)$. The corresponding symmetry current $\mathfrak{T}_{-\partial_{\Gamma}}(29)$ is the energy function $\mathcal{E}_{\Gamma}(16)$ relative to a reference frame $\Gamma^{1,5,15}$ A glance at the expression (16) shows that $\mathcal{E}_{\Gamma}=\mathcal{H}$ with respect to $\Gamma$-adapted coordinates.

Note that, given a reference frame $\Gamma$, one should solve the equations

$$
\Gamma^{i}\left(t, q^{j}\left(t, \bar{q}^{a}\right)\right)=\frac{\partial q^{i}\left(t, \bar{q}^{a}\right)}{\partial t}, \quad \frac{\partial \bar{q}^{a}\left(t, q^{j}\right)}{\partial q^{i}} \Gamma^{i}\left(t, q^{j}\right)+\frac{\partial \bar{q}^{a}\left(t, q^{j}\right)}{\partial t}=0
$$

in order to find the coordinates $\left(t, \bar{q}^{a}\right)$ adapted to $\Gamma .{ }^{1,5}$ In particular, one can show that components $\Gamma^{i}$ of $\Gamma$ are affine in coordinates $q^{i}$ iff transition functions between coordinates $q^{i}$ and $\bar{q}^{a}$ are affine, i.e., $q^{i}=a_{k}^{i}(t) \bar{q}^{k}+b^{i}(t) .{ }^{1}$ Examples in Section V illustrate this fact.

With the energy function $\mathcal{E}_{\Gamma}$, we obtain the decomposition (15) of a covariant Hamiltonian. It follows that

$$
\zeta^{*}\left(d_{t} \mathcal{E}_{\Gamma}\right) \approx\left\{\mathcal{H}^{*}, \zeta^{*} \mathcal{E}_{\Gamma}\right\}_{T}=\zeta^{*}\left(\partial_{t} \mathcal{E}_{\Gamma}\right)
$$

A Hamiltonian system is called conservative if there exists a reference frame $\Gamma$ such that, written with respect to $\Gamma$-adapted coordinates, its Hamiltonian $\mathcal{H}=\mathcal{E}_{\Gamma}$ is time-independent. A glance at the equality (31) shows that, in this case, $\mathcal{E}_{\Gamma}$ is an integral of motion, and vice versa.

Given different reference frames $\Gamma$ and $\Gamma^{\prime}$, the decomposition (15) leads at once to the relation

$$
\mathcal{E}_{\Gamma^{\prime}}=\mathcal{E}_{\Gamma}+\mathcal{H}_{\Gamma}-\mathcal{H}_{\Gamma^{\prime}}=\mathcal{E}_{\Gamma}+\left(\Gamma^{i}-\Gamma^{\prime i}\right) p_{i}
$$

\section{QUANTUM TIME-DEPENDENT MECHANICS}

Following Refs. [7-9], we quantize time-dependent mechanics in the framework of geometric quantization of the cotangent bundle $T^{*} Q$ with respect to its vertical polarization given by the vertical tangent bundle $V T^{*} Q$ of $T^{*} Q \rightarrow Q$. Note that polarization of $T^{*} Q$ need not induce polarization of $V^{*} Q$, unless it contains the vertical cotangent bundle $V_{\zeta} T^{*} Q$ of the fiber bundle $\zeta(8)$ spanned by vectors $\partial_{p}$. The above mentioned vertical polarization is unique canonical real polarization of $T^{*} Q$ satisfying this condition. With this polarization, the monomorphism of Poisson algebras

$$
\zeta^{*}:\left(C^{\infty}\left(V^{*} Q\right),\{,\}_{V}\right) \rightarrow\left(C^{\infty}\left(T^{*} Q\right),\{,\}_{T}\right)
$$


defined by the relation (10) is prolonged to a monomorphism $\mathcal{A}_{V} \rightarrow \mathcal{A}_{T}$ of quantum algebras $\mathcal{A}_{V}$ of $V^{*} Q$ and $\mathcal{A}_{T}$ of $T^{*} Q$. A result is the following (see Appendix for the quantization technique).

For the sake of simplicity, we assume that $Q$ is a simply connected manifold and that the cohomology group $H^{2}\left(Q, \mathbb{Z}_{2}\right)$ is trivial. ${ }^{9}$ Otherwise, there are nonequivalent quantizations. For the sake of convenience, the compact notation $\left(q^{\lambda}, p_{\lambda}\right), q^{0}=t, p_{0}=p$, is further used.

Quantum algebras $\mathcal{A}_{V}$ and $\mathcal{A}_{T}$ consist of affine functions of momenta on the cotangent bundle $V^{*} Q$ and the vertical cotangent bundle $T^{*} Q$, respectively. We obtain their Schrödinger representations by first order linear differential operators

$$
\begin{aligned}
& \widehat{f} \rho=\left(-i \mathbf{L}_{a^{k} \partial_{k}}+b\right) \rho=\left(-i a^{k} \partial_{k}-\frac{i}{2} \partial_{k} a^{k}+b\right) \rho, \quad f=a^{k}\left(q^{\mu}\right) p_{k}+b\left(q^{\mu}\right) \in \mathcal{A}_{V}, \\
& \widehat{f} \rho=\left(-i \mathbf{L}_{a^{\lambda} \partial_{\lambda}}+b\right) \rho=\left(-i a^{\lambda} \partial_{\lambda}-\frac{i}{2} \partial_{\lambda} a^{\lambda}+b\right) \rho, \quad f=a^{\lambda}\left(q^{\mu}\right) p_{\lambda}+b\left(q^{\mu}\right) \in \mathcal{A}_{T},
\end{aligned}
$$

which act in the $C^{\infty}(\mathbb{R})$-module $E_{R}$ of complex half-forms $\rho$ on $Q$ whose restriction to each fiber $Q_{t}$ of $Q \rightarrow \mathbb{R}$ is of compact support. A glance at the expressions (33) and (34) shows that $\widehat{f}$ (33) is the representation of $\mathcal{A}_{V}$ as a subalgebra of $\mathcal{A}_{T}$.

It is readily justified that the operators (33) and (34) satisfy the Dirac condition

$$
\left[\widehat{f}, \widehat{f}^{\prime}\right]=-i\left\{\widehat{f, f^{\prime}}\right\}_{T}
$$

and, thus, form real Lie algebras. Moreover, the quantum operators of functions $f(t) \in$ $C^{\infty}(\mathbb{R})$ depending only on time are reduced to multiplications $\widehat{f} \rho=f \rho$, and they commute with any element $(33)$ of the quantum algebra $\mathcal{A}_{V}$. It follows that $\mathcal{A}_{V}$ is a Lie $C^{\infty}(\mathbb{R})$ algebra. Its representation (33) defines the instantwise quantization of $\mathcal{A}_{V}$ as follows.

Geometric quantization of the Poisson manifold $\left(V^{*} Q,\{,\}_{V}\right)$ yields geometric quantization of its symplectic leaves which are fibers $i_{t}: V_{t}^{*} Q \rightarrow V^{*} Q, t \in \mathbb{R}$ of the fiber bundle $V^{*} Q \rightarrow \mathbb{R}$ provided with the symplectic structure

$$
\Omega_{t}=\left(\mathcal{H} \circ i_{t}\right)^{*} \Omega=d p_{k} \wedge d q^{k}
$$

where $\mathcal{H}$ is an arbitrary section of the fiber bundle $\zeta(8)$. The associated quantum algebra $\mathcal{A}_{t}$ consists of functions on $V_{t}^{*} Q$ which are affine in momenta. It is represented by Hermitian operators

$$
\widehat{f}_{t} \rho_{t}=\left(-i \mathbf{L}_{a^{k} \partial_{k}}+b\right) \rho_{t}=\left(-i a^{k} \partial_{k}-\frac{i}{2} \partial_{k} a^{k}+b\right) \rho_{t}, \quad f_{t}=a^{k}\left(q^{i}\right) p_{k}+b\left(q^{i}\right) \in \mathcal{A}_{t},
$$

in the pre-Hilbert space $E_{t}$ of half-forms of compact support on $Q_{t}$ equipped with the Hermitian metric

$$
\left\langle\rho_{t}^{\prime} \mid \rho_{t}\right\rangle_{t}=\int_{Q_{t}} \bar{\rho}_{t}^{\prime} \rho_{t} .
$$


Spaces $E_{t}, t \in \mathbb{R}$, are assembled into a trivial pre-Hilbert bundle over $\mathbb{R}$. One can show that the restriction to $V_{t}^{*} Q$ of any element of the quantum algebra $\mathcal{A}_{V}$ belongs to $\mathcal{A}_{t}$ and, conversely, any element of $\mathcal{A}_{t}$ is of this type. Thus, $\mathcal{A}_{t}=i_{t}^{*} \mathcal{A}_{V}$. Any half-form $\rho \in E_{R}$ on $Q$ yields a half-form of compact support on $Q_{t}$. Given an element $f \in \mathcal{A}_{V}$ and its pull-back $f_{t}=i_{t}^{*} f \in \mathcal{A}_{t}$, we obtain from the formulas (33) and (37) that $\widehat{f} \rho \circ i_{t}=\widehat{f}_{t}\left(\rho \circ i_{t}\right)$.

As was mentioned above, the $C^{\infty}(\mathbb{R})$-module $E_{R}$ is also the carrier space for the quantum algebra $\mathcal{A}_{T}$, but its action on $E_{R}$ is not instantwise.

\section{ENERGY OPERATORS}

Turn now to quantization of the covariant Hamiltonian $\mathcal{H}^{*}(13)$. A problem is that, in the framework of the Schrödinger quantization, it fails to belong to the quantum algebra $\mathcal{A}_{T}$, unless it is affine in momenta. Let us restrict our consideration to the physically relevant case of $\mathcal{H}^{*}$ polynomial in momenta. One can show that, in this case, $\mathcal{H}^{*}$ is decomposed in a finite sum of products of elements of the algebra $\mathcal{A}_{T}$, though this decomposition by no means is unique. ${ }^{7,9}$ Provided with such a decomposition, $\mathcal{H}^{*}$ is an element of the universal enveloping algebra $\overline{\mathcal{A}}_{T}$ of the Lie algebra $\mathcal{A}_{T}$. To be more precise, it belongs to $\mathcal{A}_{T}+\overline{\mathcal{A}}_{V}$, where $\overline{\mathcal{A}}_{V}$ is the universal enveloping algebra of the Lie algebra $\mathcal{A}_{V} \subset \mathcal{A}_{T}$. Since the Dirac condition (35) holds, the Schrödinger representation of the Lie algebras $\mathcal{A}_{T}$ and $\mathcal{A}_{V}$ is naturally extended to their enveloping algebras $\overline{\mathcal{A}}_{T}$ and $\overline{\mathcal{A}}_{V}$, and provides the quantization $\widehat{\mathcal{H}}^{*}$ of a covariant Hamiltonian $\mathcal{H}^{*}$.

For instance, let $\mathcal{H}_{\Gamma}(12)$ be a Hamiltonian defined by a connection $\Gamma$ on $V^{*} Q$. The corresponding covariant Hamiltonian

$$
\mathcal{H}_{\Gamma}^{*}=p+\mathcal{H}_{\Gamma}=p+\Gamma^{k} p_{k}
$$

belongs to the quantum algebra $\mathcal{A}_{T}$. Its quantization (33) reads

$$
\widehat{\mathcal{H}}_{\Gamma}^{*}=-i \partial_{t}-i \Gamma^{k} \partial_{k}-\frac{i}{2} \partial_{k} \Gamma^{k}
$$

Written with respect to $\Gamma$-adapted coordinates, it takes the form $\widehat{\mathcal{H}}_{\Gamma}^{*}=-i \partial_{t}$.

Given an operator $\widehat{\mathcal{H}}^{*}$, the bracket

$$
\widehat{\nabla} \widehat{f}=i\left[\widehat{\mathcal{H}}^{*}, \widehat{f}\right]
$$

defines a derivation of the algebra $\overline{\mathcal{A}}_{V}$. Moreover, since $\widehat{p}=-i \partial_{t}$, the derivation (41) obeys the Leibniz rule

$$
\widehat{\nabla}(g \widehat{f})=\partial_{t} g \widehat{f}+g \nabla \widehat{f}, \quad g \in C^{\infty}(\mathbb{R})
$$


Therefore, it is a connection on the $C^{\infty}(\mathbb{R})$-module $\overline{\mathcal{A}}_{V} \cdot{ }^{5,9}$ One says that $\widehat{f}$ is parallel with respect to the connection (41) if

$$
\left[\widehat{\mathcal{H}}^{*}, \widehat{f}\right]=0 .
$$

One can think of this equality as being the Heisenberg equation in time-dependent mechanics, while the quantum constraint

$$
\widehat{\mathcal{H}}^{*} \rho=0, \quad \rho \in E_{R},
$$

plays a role of the Schrödinger equation.

For instance, any connection (i.e., a reference frame) $\Gamma$ (2) on a configuration bundle $Q \rightarrow \mathbb{R}$ induces the connection

$$
\widehat{\nabla}_{\Gamma} \widehat{f}=i\left[\widehat{\mathcal{H}}_{\Gamma}, \widehat{f}\right]
$$

on the algebra $\overline{\mathcal{A}}_{V}$ which is also a connection on the quantum algebra $\mathcal{A}_{V} \subset \overline{\mathcal{A}}_{V}$. The corresponding Schrödinger equation (43) reads

$$
-i\left(\partial_{t}+\Gamma^{k} \partial_{k}+\frac{1}{2} \partial_{k} \Gamma^{k}\right) \rho=0
$$

Its solutions are half-forms $\rho \in E_{R}$ which, written relative to $\Gamma$-adapted coordinates $\left(t, \bar{q}^{j}\right)$, are time-independent, i.e., $\rho=\rho\left(\bar{q}^{j}\right)$.

Given a reference frame $\Gamma$, the energy function $\mathcal{E}_{\Gamma}$ is quantized as $\widehat{\mathcal{E}}_{\Gamma}=\widehat{\mathcal{H}}^{*}-\widehat{\mathcal{H}}_{\Gamma}^{*}$. One can think of $\widehat{\mathcal{E}}_{\Gamma}$ as being an operator of energy with respect to the reference frame $\Gamma$. As a consequence, the Schrödinger equation (43) reads

$$
\left(\widehat{\mathcal{H}}_{\Gamma}+\widehat{\mathcal{E}}_{\Gamma}\right) \rho=-i\left(\partial_{t}+\Gamma^{k} \partial_{k}+\frac{1}{2} \partial_{k} \Gamma^{k}\right) \rho+\widehat{\mathcal{E}}_{\Gamma} \rho=0 .
$$

Let a Hamiltonian system be conservative and $\Gamma$ a reference frame such that the energy function $\mathcal{E}_{\Gamma}$ is time-independent relative to $\Gamma$-adapted coordinates. In this case, the Schrödinger equation (45) takes the familiar form

$$
\left(-i \partial_{t}+\widehat{\mathcal{E}}_{\Gamma}\right) \rho=0
$$

It follows from the Heisenberg equation (42) that a Hamiltonian system is conservative iff there exists a reference frame $\Gamma$ such that

$$
\left[\widehat{\mathcal{H}}^{*}, \widehat{\mathcal{E}}_{\Gamma}\right]=0
$$

Given different reference frames, operators of energy $\widehat{\mathcal{E}}_{\Gamma}$ and $\widehat{\mathcal{E}}_{\Gamma^{\prime}}$ obey the relation (18) taking the form

$$
\widehat{\mathcal{E}}_{\Gamma^{\prime}}=\widehat{\mathcal{E}}_{\Gamma}-i\left(\Gamma^{k}-\Gamma^{\prime k}\right) \partial_{k}-\frac{i}{2} \partial_{k}\left(\Gamma^{k}-\Gamma^{\prime k}\right) .
$$


In particular, let $\widehat{\mathcal{E}}_{\Gamma}$ be a time-independent energy operator of a conservative system, and let $\rho_{E}$ be its eigenstate of eigenvalue $E$, i.e., $\widehat{\mathcal{E}}_{\Gamma} \rho_{E}=E \rho_{E}$. Then the energy of this state relative to a reference frame $\Gamma^{\prime}$ at an instant $t$ is

$$
\begin{array}{r}
\left\langle\rho_{E} \mid \widehat{\mathcal{E}}_{\Gamma^{\prime}} \rho_{E}\right\rangle=E+i\left\langle\rho_{E} \mid\left(\Gamma^{\prime k} \partial_{k}+\frac{1}{2} \partial_{k} \Gamma^{\prime k}\right) \rho_{E}\right\rangle_{t}= \\
E+i \int_{Q_{t}} \bar{\rho}_{E}\left(\Gamma^{\prime k}\left(q^{j}, t\right) \partial_{k}+\frac{1}{2} \partial_{k} \Gamma^{\prime k}\left(q^{j}, t\right)\right) \rho_{E} .
\end{array}
$$

\section{EXAMPLES}

Let us consider a Hamiltonian system on $Q=\mathbb{R} \times U$, where $U \subset \mathbb{R}^{m}$ is an open domain equipped with coordinates $\left(q^{i}\right)$. These coordinates yield a reference frame on $Q$ given by the connection $\Gamma$ such that $\Gamma^{i}=0$ with respect to these coordinates. Let it be a conservative Hamiltonian system whose energy function $\mathcal{E}_{\Gamma}$, written relative to coordinates $\left(t, q^{i}\right)$, is time-independent. Let us consider a different reference frame on $Q$ given by the connection

$$
\Gamma^{\prime}=d t \otimes\left(\partial_{t}+G^{i} \partial_{i}\right), \quad G^{i}=\text { const }
$$

on $Q$. The $\Gamma^{\prime}$-adapted coordinates $\left(t, q^{\prime j}\right)$ obey the equations (30) which read

$$
G^{i}=\frac{\partial q^{i}\left(t, q^{\prime j}\right)}{\partial t}, \quad \frac{\partial q^{\prime j}\left(t, q^{i}\right)}{\partial q^{k}} G^{k}+\frac{\partial q^{\prime j}\left(t, q^{i}\right)}{\partial t}=0 .
$$

We obtain $q^{i}=q^{i}-G^{i} t$. For instance, this is the case of inertial frames. ${ }^{1}$ Given by the relation (32), the energy function relative to the reference frame $\Gamma^{\prime}$ (48) reads

$$
\mathcal{E}_{\Gamma^{\prime}}=\mathcal{E}_{\Gamma}-G^{k} p_{k}
$$

Accordingly, the relation (47) between operators of energy $\widehat{\mathcal{E}}_{\Gamma^{\prime}}$ and $\widehat{\mathcal{E}}_{\Gamma}$ takes the form

$$
\widehat{\mathcal{E}}_{\Gamma^{\prime}}=\widehat{\mathcal{E}}_{\Gamma}+i G^{k} \partial_{k}
$$

Let $\rho_{E}$ be an eigenstate of the energy operator $\widehat{\mathcal{E}}_{\Gamma}$. Then its energy with respect to the reference frame $\Gamma^{\prime}(48)$ is $E-G^{k} P_{k}$, where $P_{k}=\left\langle\rho_{E} \mid \widehat{p}_{k} \rho_{E}\right\rangle_{t}$ are momenta of this state. This energy is time-independent.

In particular, the following condition holds in many physical models. Given an eigenstate $\rho_{E}$ of the energy operator $\widehat{\mathcal{E}}_{\Gamma}$ and a reference frame $\Gamma^{\prime}(48)$, there is the equality

$$
\widehat{\mathcal{E}}_{\Gamma^{\prime}}\left(\widehat{p}^{j}, q^{j}\right) \rho_{E}=\left(\widehat{\mathcal{E}}_{\Gamma}\left(\widehat{p}^{j}, q^{j}\right)-G^{k}\left(q^{j}\right) \widehat{p}_{k}\right) \rho_{E}=\left(\widehat{\mathcal{E}}_{\Gamma}\left(\widehat{p}^{j}+A_{j}, q^{j}\right)+B\right) \rho_{E}, \quad A_{j}, B=\mathrm{const} .
$$

Then $\exp \left(-i A_{j} q^{j}\right) \rho_{E}$ is an eigenstate of the energy operator $\widehat{\mathcal{E}}_{\Gamma^{\prime}}$ possessing the eigenvalue $E+B$. 
For instance, any Hamiltonian

$$
\widehat{\mathcal{H}}=\widehat{\mathcal{E}}_{\Gamma}=\frac{1}{2}\left(m^{-1}\right)^{i j} \widehat{p}_{i} \circ \widehat{p}_{j}+V\left(q^{j}\right)
$$

quadratic in momenta $\widehat{p}_{i}$ with a nondegenerate constant mass tensor $m^{i j}$ obeys this condition. ${ }^{1}$ Namely, we have

$$
A_{i}=-m_{i j} G^{j}, \quad B=-\frac{1}{2} m_{i j} G^{i} G^{j}
$$

Let us consider a massive point particle in an Euclidean space $\mathbb{R}^{3}$ in the presence of a central potential $V(r)$. Let $\mathbb{R}^{3}$, be equipped with the spherical coordinates $(r, \phi, \theta)$. These coordinates define an inertial reference frame $\Gamma$ such that $\Gamma^{r}=\Gamma^{\phi}=\Gamma^{\theta}=0$. The Hamiltonian of the above mentioned particle with respect to this reference frame reads

$$
\widehat{\mathcal{H}}=\widehat{\mathcal{E}}_{\Gamma}=\frac{1}{m}\left(-\frac{1}{r} \partial_{r}-\frac{1}{2} \partial_{r}^{2}+\frac{\widehat{I}^{2}}{r^{2}}\right)+V(r),
$$

where $\widehat{I}$ is the square of the angular momentum operator. Let us consider a rotatory reference frame $\Gamma^{\prime \phi}=\omega, \omega=$ const, given by the adapted coordinates $\left(r, \phi^{\prime}=\phi-\omega t, \theta\right)$. The operator of energy relative to this reference frame is

$$
\widehat{\mathcal{E}}_{\Gamma^{\prime}}=\widehat{\mathcal{E}}_{\Gamma}+i \omega \partial_{\phi}
$$

Let $\rho_{E, n, l}$ be an eigenstate of the energy operator $\widehat{\mathcal{E}}_{\Gamma}(51)$ posessing its eigenvalue $E$, the eigenvalue $n$ of the angular momentum operator $\widehat{I}_{3}=\widehat{p}_{\phi}$, and the eigenvalue $l(l+1)$ of the operator $\widehat{I}^{2}$. Then $\rho_{E, n, l}$ is also an eigenstate of the energy operator $\widehat{\mathcal{E}}_{\Gamma^{\prime}}$ with the eigenvalue $E^{\prime}=E-n \omega$.

\section{APPENDIX}

We start with the standard prequantization of the cotangent bundle $T^{*} Q$. Since the symplectic form $\Omega(6)$ on $T^{*} Q$ is exact, a prequantization bundle is the trivial complex line bundle

$$
C=T^{*} Q \times \mathbb{C} \rightarrow T^{*} Q,
$$

coordinated by $\left(q^{\lambda}, p_{\lambda}, c\right)$. It is provided with the admissible linear connection

$$
A=d p_{\lambda} \otimes \partial^{\lambda}+d q^{\lambda} \otimes\left(\partial_{\lambda}+i p_{\lambda} c \partial_{c}\right)
$$

whose curvature equals $-i \Omega$. The $A$-invariant Hermitian fiber metric on $C$ is $g\left(c, c^{\prime}\right)=c \bar{c}^{\prime}$. The covariant derivative of sections $s$ of the prequantization bundle $C$ (53) relative to the connection $A$ (54) along a vector field $u$ on $T^{*} Q$ reads

$$
\nabla_{u} s=u^{\lambda}\left(\partial_{\lambda}-i p_{\lambda}\right) s+u_{\lambda} \partial^{\lambda} s .
$$


Given a function $f \in C^{\infty}\left(T^{*} Q\right)$ and its Hamiltonian vector field $u_{f}=\partial^{\lambda} f \partial_{\lambda}-\partial_{\lambda} f \partial^{\lambda}$, one assigns to $f$ the first order differential operator

$$
\widehat{f}(s)=-i\left(\nabla_{u_{f}}+i f\right) s=\left[-i\left(\partial^{\lambda} f \partial_{\lambda}-\partial_{\lambda} f \partial^{\lambda}\right)+\left(f-p_{\lambda} \partial^{\lambda} f\right)\right] s
$$

on sections $s$ of the prequantization bundle $C(53)$. These operators obey the Dirac condition (35) and, thus, form a prequantum real Lie algebra $\widehat{C}^{\infty}\left(T^{*} Q\right)$ of $T^{*} Q$.

Let us turn to prequantization of the Poisson manifold $\left(V^{*} Q,\{,\}_{V}\right)$. Its Poisson bivector field $w=\partial^{k} \wedge \partial_{k}$ is exact. Therefore, a prequantization bundle is the trivial complex line bundle

$$
C_{V}=V^{*} Q \times \mathbb{C} \rightarrow V^{*} Q
$$

Since the bundles $C(53)$ and $C_{V}$ (56) are trivial, $C$ can be seen as the pull-back $\zeta^{*} C_{V}$ of $C_{V}$, while $C_{V}$ is isomorphic to the pull-back $\mathcal{H}^{*} C$ of $C$ with respect to some section $\mathcal{H}$ (11) of the affine bundle (8). Because the covariant derivative of the connection $A(54)$ along the fibers of $\zeta(8)$ is trivial, let us consider the pull-back

$$
\mathcal{H}^{*} A=d p_{k} \otimes \partial^{k}+d q^{k} \otimes\left(\partial_{k}+i p_{k} c \partial_{c}\right)+d t \otimes\left(\partial_{t}-i \mathcal{H} c \partial_{c}\right)
$$

of $A$ onto $C_{V} \rightarrow V^{*} Q$. This connection defines the contravariant derivative

$$
\nabla_{\phi} s_{V}=\nabla_{w^{\sharp} \phi} s_{V}, \quad w^{\sharp} \phi=\phi^{k} \partial_{k}-\phi_{k} \partial^{k},
$$

of sections $s_{V}$ of $C_{V} \rightarrow V^{*} Q$ along one-forms $\phi$ on $V^{*} Q$. The contravariant derivative (58) corresponds to a contravariant connection $A_{V}$ on the line bundle $C_{V} \rightarrow V^{*} Q .{ }^{16}$ Since vector fields $w^{\sharp} \phi$ are vertical on $V^{*} Q \rightarrow \mathbb{R}$, this contravariant connection does not depend on a choice of $\mathcal{H}$. By virtue of the relation (58), the curvature bivector of $A_{V}$ equals $-i w$, i.e., $A_{V}$ is an admissible connection on $V^{*} Q$. Then the Kostant-Souriau formula

$$
\widehat{f}\left(s_{V}\right)=\left(-i \nabla_{\vartheta_{f}}+f\right) s_{V}=\left[-i\left(\partial^{k} f \partial_{k}-\partial_{k} f \partial^{k}\right)+\left(f-p_{k} \partial^{k} f\right)\right] s_{V}
$$

defines prequantization of the Poisson manifold $V^{*} Q$. In particular, the prequantum operators of functions $f(t) \in C^{\infty}(\mathbb{R})$ of time are reduced to multiplications $\widehat{f}\left(s_{V}\right)=f s_{V}$, and they commute with any element (59) of the quantum algebra $\mathcal{A}_{V}$. Consequently, the prequantum algebra $\widehat{C}^{\infty}\left(V^{*} Q\right)$ of $V^{*} Q$ is a Lie $C^{\infty}(\mathbb{R})$-algebra. It is readily observed that the prequantum operator $\widehat{f}(59)$ coincides with the prequantum operator $\widehat{\zeta^{*} f}(55)$ restricted to the pull-back sections $s=\zeta^{*} s_{V}$. Thus, the above mentioned prequantization of the Poisson algebra $C^{\infty}\left(V^{*} Q\right)$ is equivalent to its prequantization as a subalgebra of the Poisson algebra $C^{\infty}\left(T^{*} Q\right)$. Since the line bundles $C(53)$ and $C_{V}(56)$ are trivial, their sections are smooth complex functions on $T^{*} Q$ and $V^{*} Q$, respectively. Then the prequantum operators (55) and (59) can be written in the form

$$
\widehat{f}=-i \mathbf{L}_{u_{f}}+\left(f-\mathbf{L}_{\vartheta} f\right),
$$


where $\vartheta$ is either the Liouville vector field $\vartheta=p_{\lambda} \partial^{\lambda}$ on $T^{*} Q$ or $\vartheta=p_{k} \partial^{k}$ on $V^{*} Q$.

Given compatible prequantizations of $T^{*} Q$ and $V^{*} Q$, let us construct their compatible polarizations and quantizations. Recall that polarization of a Poisson manifold $(Z,\{\}$, is defined as a sheaf $\mathbf{T}^{*}$ of germs of complex functions on $Z$ whose stalks $\mathbf{T}_{z}^{*}, z \in Z$, are Abelian algebras with respect to the Poisson bracket $\{,\} .{ }^{16,17}$. A quantum algebra $\mathcal{A}$ associated to the Poisson polarization $\mathbf{T}^{*}$ is a subalgebra of the Poisson algebra $C^{\infty}(Z)$ which consists of functions $f$ such that $\left\{f, \mathbf{T}^{*}(Z)\right\} \subset \mathbf{T}^{*}(Z)$. Polarization of a symplectic manifold yields its Poisson one.

Let $\mathbf{T}^{*}$ be a polarization of the Poisson manifold $\left(T^{*} Q,\{,\}_{T}\right)$. Its direct image in $V^{*} Q$ with respect to the fibration $\zeta(8)$ is polarization of the Poisson manifold $\left(V^{*} Q,\{,\}_{V}\right)$ if elements of $\mathbf{T}^{*}$ are germs of functions constant on fibers of $\zeta$, i.e., independent of the momentum coordinate $p$. It follows that the corresponding symplectic polarization $\mathbf{T}$ of $T^{*} Q$ is vertical with respect to $\zeta$. The polarization $\mathbf{T}=V T^{*} Q$ of $T^{*} Q$ obeys this condition. The associated quantum algebra $\mathcal{A}_{T} \subset C^{\infty}\left(T^{*} Q\right)$ consists of functions which are affine in momenta $p_{\lambda}$. It acts by operators (60) on the space of smooth complex functions $s$ on $T^{*} Q$ which fulfill the relation $\nabla_{u} s=0$ for any vertical vector field $u$ on $T^{*} Q \rightarrow Q$. Clearly, these functions are the pull-back of complex functions on $Q$ along the fibration $T^{*} Q \rightarrow Q$. Following the metaplectic correction procedure, we come to complex half-forms on $Q$ which are sections of the complex line bundle $\mathcal{D}_{1 / 2} \rightarrow Q$ with the transition functions $c^{\prime}=S c$ such that $S \bar{S}$ is the Jacobian of coordinate transition functions on $Q$. Then the formula (60), where $\mathbf{L}$ is the Lie derivative of half-forms, defines the Schrödinger representation of the real Lie algebra $\mathcal{A}_{T}$ by operators (34) in the space $\mathcal{D}_{1 / 2}(Q)$ of complex half-forms $\rho$ on $Q$. These operators are Hermitian on the pre-Hilbert space $E \subset \mathcal{D}_{1 / 2}(Q)$ of half-forms of compact support provided with the nondegenerate Hermitian form

$$
\left\langle\rho^{\prime} \mid \rho\right\rangle=\int_{Q} \bar{\rho}^{\prime} \rho .
$$

The vertical polarization of $T^{*} Q$ induces the polarization $\mathbf{T}_{V}^{*}$ of the Poisson manifold $V^{*} Q$ which contains the germs of functions constant on fibers of $V^{*} Q \rightarrow Q$. The associated quantum algebra $\mathcal{A}_{V}$ consists of functions on $V^{*} Q$ which are affine in momenta. It acts by operators (60) on the space of smooth complex functions $s_{V}$ on $V^{*} Q$ which fulfill the relation $\nabla_{u} s_{V}=0$ for any vertical vector field $u$ on $V^{*} Q \rightarrow Q$. These functions are the pull-back of complex functions on $Q$ along the fibration $V^{*} Q \rightarrow Q$. Similarly to the case of $\mathcal{A}_{T}$, we obtain the Schrödinger representation of $\mathcal{A}_{V}$ by the operators (33) on half-forms on $Q$. Moreover, a glance at the expressions (33) and (34) shows that (33) is the representation of $\mathcal{A}_{V}$ as a subalgebra of $\mathcal{A}_{T}$.

However, the physical relevance of the pre-Hilbert space $E$ with the Hermitian form (61) is open to question. This Hermitian form implies integration over time, though the 
time plays a role of the classical evolution parameter in quantum mechanics. Since the representation (33) preserves the structure of $\mathcal{A}_{V}$ as a Lie $C^{\infty}(\mathbb{R})$-algebra, let us show that this representation defines the instantwise quantization of $\mathcal{A}_{V}$.

The prequantization (59) of the Poisson manifold $V^{*} Q$ yields prequantization of its symplectic leaf $i_{t}: V_{t}^{*} Q \rightarrow V^{*} Q, t \in \mathbb{R}$, endowed with the symplectic form (36). Since $w^{\sharp} \phi$ is a vertical vector field on $V^{*} Q \rightarrow \mathbb{R}$ for any one-form $\phi$ on $V^{*} Q$, the contravariant derivative (58) defines a connection along each fiber $V_{t}^{*} Q, t \in \mathbb{R}$, of the Poisson bundle $V^{*} Q \rightarrow \mathbb{R}$. It is the pull-back

$$
A_{t}=i_{t}^{*} h^{*} A=d p_{k} \otimes \partial^{k}+d q^{k} \otimes\left(\partial_{k}+i p_{k} c \partial_{c}\right)
$$

of the connection $\mathcal{H}^{*} A(57)$ onto the trivial pull-back line bundle

$$
i_{t}^{*} C_{V}=V_{t}^{*} Q \times \mathbb{C} \rightarrow V_{t}^{*} Q
$$

It is readily observed that this connection is admissible for the symplectic structure (36) on $V_{t}^{*} Q$, and provides prequantization of the symplectic manifold $\left(V_{t}^{*} Q, \Omega_{t}\right)$ by the formula

$$
\widehat{f}_{t}=-i \mathbf{L}_{\vartheta_{f_{t}}}+\left(f_{t}-\mathbf{L}_{\vartheta_{t}}\right)=-i\left(\partial^{k} f_{t} \partial_{k}-\partial_{k} f_{t} \partial^{k}\right)+\left(f_{t}-p_{k} \partial^{k} f_{t}\right),
$$

where $\vartheta_{f_{t}}=\partial^{k} f_{t} \partial_{k}-\partial_{k} f_{t} \partial^{k}$ is the Hamiltonian vector field of a function $f_{t}$ on $V_{t}^{*} Q$ with respect to the symplectic form $\Omega_{t}(36)$. The operators (62) act on smooth complex functions $s_{t}$ on $V_{t}^{*} Q$. In particular, let $f_{t}, s_{t}$ and $(\widehat{f} s)_{t}$ be the restriction to $V_{t}^{*} Q$ of a real function $f$ and complex functions $s$ and $\widehat{f}(s)$ on $V^{*} Q$, respectively. We obtain from the formulas (59) and (62) that $(\widehat{f} s)_{t}=\widehat{f}_{t} s_{t}$. It follows that the prequantization (59) of the Poisson manifold $V^{*} Q$ is a fiberwise prequantization.

Let $\mathbf{T}_{V}^{*}$ be the above mentioned polarization of the Poisson manifold $V^{*} Q$. It yields the pull-back polarization $\mathbf{T}_{t}^{*}=i_{t}^{*} \mathbf{T}_{V}^{*}$ of a fiber $V_{t}^{*} Q$ with respect to the Poisson morphism $i_{t}: V_{t}^{*} Q \rightarrow V^{*} Q$. The corresponding distribution $\mathbf{T}_{t}$ coincides with the vertical tangent bundle of the fiber bundle $V_{t}^{*} Q \rightarrow Q_{t}$. The associated quantum algebra $\mathcal{A}_{t}$ consists of functions on $V^{*} Q_{t}$ which are affine in momenta. In particular, the restriction to $V_{t}^{*} Q$ of any element of the quantum algebra $\mathcal{A}_{V}$ obeys this condition and, consequently, belongs $\mathcal{A}_{t}$. Conversely, any element of $\mathcal{A}_{t}$ is of this type. ${ }^{7,9}$ Thus, $\mathcal{A}_{t}=i_{t}^{*} \mathcal{A}_{V}$ and, therefore, the polarization $\mathbf{T}_{V}^{*}$ of the Poisson bundle $V^{*} Q \rightarrow \mathbb{R}$ is a fiberwise polarization.

In order to provide metaplectic correction and to complete geometric quantization of symplectic fibers of the Poisson bundle $V^{*} Q \rightarrow \mathbb{R}$, we use the fact that the Jacobian $J$ of the transition function between bundle coordinates $\left(t, q^{k}\right)$ and $\left(t, q^{\prime k}\right)$ on $Q$ coincides with the Jacobian $J_{t}$ of the transition function between coordinates $\left(q^{k}\right)$ and $\left(q^{\prime k}\right)$ on the fiber $Q_{t}$ of $Q$ over a point $t$. One can show that, given a bundle $\mathcal{D}_{1 / 2} \rightarrow Q$ of complex half-forms on $Q$, its pull-back $i_{t}^{*} \mathcal{D}_{1 / 2}$ onto $Q_{t}$ is the fiber bundle of half-forms on $Q_{t} \cdot{ }^{7,9}$ Then the formula 
(62) defines the Schrödinger representation of the quantum algebra $\mathcal{A}_{t}$ of the symplectic fiber $Q_{t}$ by Hermitian operators (37) in the pre-Hilbert space $E_{t}$ of half-forms x of compact support on $Q_{t}$ equipped with the Hermitian metric (38). Spaces $E_{t}, t \in \mathbb{R}$, are assembled into a trivial pre-Hilbert bundle over $\mathbb{R}$.

Any half-form $\rho$ on $Q$ yields a half-form on $Q_{t}$. Given an element $f \in \mathcal{A}_{V}$ and its pull-back $f_{t}=i_{t}^{*} f \in \mathcal{A}_{t}$, we obtain from the formulae (33) and (37) that $\hat{f} \rho \circ i_{t}=\widehat{f}_{t}\left(\rho \circ i_{t}\right)$. This equality shows that the Schrödinger quantization of the Poisson manifold $V^{*} Q$ can be seen as the instantwise quantization.

1 L.Mangiarotti and G.Sardanashvily, Gauge Mechanics (World Scientific, Singapore, 1998).

2 G.Sardanashvily, J. Math. Phys. 39, 2714 (1998); E-print arXiv: dg-ga/9710003.

3 L.Mangiarotti and G.Sardanashvily, J. Math. Phys. 41, 2858 (2000); E-print arXiv: math-ph/9904028.

4 E.Massa and E.Pagani, Ann. Inst. Henri Poincaré 61, 17 (1994).

5 L.Mangiarotti and G.Sardanashvily, Connections in Classical and Quantum Field Theory (World Scientific, Singapore, 2000).

6 D.Canarutto, Bull. U.M.I. Algebra e Geometria, Serie VI V-D, 18 (1986).

7 G.Giachetta, L.Mangiarotti and G.Sardanashvily, J. Math. Phys. 43, 56 (2002); Eprint arXiv: quant-ph/0012036.

8 G.Giachetta, L.Mangiarotti and G.Sardanashvily, J. Math. Phys. 43, 2882 (2002); E-print arXiv: quant-ph/0112011.

9 G.Giachetta, L.Mangiarotti and G.Sardanashvily, Geometric and Algebraic Topological Methods in Quantum Mechanics (World Scientific, Singapore, 2005).

10 J.Śniatycki, Geometric Quantization and Quantum Mechanics (Springer, Berlin, 1980).

11 N.Woodhouse, Geometric Quantization (Clarendon Press, Oxford, 1992).

12 A.Echeverría Enríquez, M.Muñoz Lecanda and N.Román Roy, J. Phys. A 28, 5553 (1995). 
13 A.Lichtenberg and M.Liebermann, Regular and Stochastic Motion (Springer, Berlin, 1983).

14 A.Dewisme and S.Bouquet, J. Math. Phys. 34, 997 (1993).

15 G.Sardanashvily, J. Math. Phys. 38, 847 (1997); E-print arXiv: gr-qc/9503038.

16 I.Vaisman, Lectures on the Geometry of Poisson Manifolds (Birkhäuser Verlag, Basel, 1994).

17 I.Vaisman, Diff. Geom. Appl. 7, 265 (1997). 